\title{
Estimation under matrix quadratic loss and matrix superharmonicity
}

\author{
Takeru Matsuda* and William E. Strawderman ${ }^{\dagger}$
}

\begin{abstract}
We investigate estimation of a normal mean matrix under the matrix quadratic loss. Improved estimation under the matrix quadratic loss implies improved estimation of any linear combination of the columns. First, an unbiased estimate of risk is derived and the Efron-Morris estimator is shown to be minimax. Next, a notion of matrix superharmonicity for matrix-variate functions is introduced and shown to have analogous properties with usual superharmonic functions, which may be of independent interest. Then, we show that the generalized Bayes estimator with respect to a matrix superharmonic prior is minimax. We also provide a class of matrix superharmonic priors that includes the previously proposed generalization of Stein's prior. Numerical results demonstrate that matrix superharmonic priors work well for low rank matrices.
\end{abstract}

\section{Introduction}

Suppose that we have a matrix observation $X \in \mathbb{R}^{n \times p}$ whose entries are independent normal random variables $X_{i j} \sim \mathrm{N}\left(M_{i j}, 1\right)$, where $n-p-1>0$ and $M \in \mathbb{R}^{n \times p}$ is an unknown mean matrix. By using the notation of matrix-variate normal distributions, it is expressed as $X \sim \mathrm{N}_{n, p}\left(M, I_{n}, I_{p}\right)$, where $I_{n}$ is the $n$-dimensional identity matrix. In this setting, we consider estimation of $M$ under the matrix quadratic loss [1, 3, 10, 18]:

$$
L(M, \hat{M})=(\hat{M}-M)^{\top}(\hat{M}-M),
$$

which takes a value in the set of $p \times p$ positive semidefinite matrices. Namely, the risk function of an estimator $\hat{M}=\hat{M}(X)$ is defined as

$$
R(M, \hat{M})=\mathrm{E}_{M}[L(M, \hat{M}(X))],
$$

and an estimator $\hat{M}_{1}$ is said to dominate another estimator $\hat{M}_{2}$ if $R\left(M, \hat{M}_{1}\right) \preceq R\left(M, \hat{M}_{2}\right)$ for every $M$, where $\preceq$ is the Löwner order: $A \preceq B$ means that $B-A$ is positive semidefinite. Thus, if $\hat{M}_{1}$ dominates $\hat{M}_{2}$, then

$$
\mathrm{E}_{M}\left[\left\|\left(\hat{M}_{1}-M\right) c\right\|^{2}\right] \leq \mathrm{E}_{M}\left[\left\|\left(\hat{M}_{2}-M\right) c\right\|^{2}\right]
$$

for every $M$ and $c \in \mathbb{R}^{p}$. In particular, each column of $\hat{M}_{1}$ dominates that of $\hat{M}_{2}$ as an estimator of the corresponding column of $M$ under quadratic loss.

\footnotetext{
${ }^{*}$ RIKEN Center for Brain Science, e-mail: takeru.matsuda@riken.jp

${ }^{\dagger}$ Department of Statistics and Biostatistics, Rutgers University
} 
In the context of multivariate linear regression, improved estimation of the regression coefficient matrix under the matrix quadratic loss implies improved estimation of mean response for any value of the explanatory variables. Specifically, let $x_{i} \in \mathbb{R}^{p}$ and $y_{i} \sim \mathrm{N}_{q}\left(B^{\top} x_{i}, \Sigma\right)$ for $i=1, \ldots, n$, where $B \in \mathbb{R}^{p \times q}$ and $\Sigma \in \mathbb{R}^{q \times q}$ is a known covariance matrix. By using the notation of matrix-variate normal distributions, it is expressed as $Y \sim \mathrm{N}_{n, q}\left(X B, I_{n}, \Sigma\right)$, where $X=\left(x_{1}, \ldots, x_{n}\right)^{\top} \in \mathbb{R}^{n \times p}$ and $Y=\left(y_{1}, \ldots, y_{n}\right)^{\top} \in \mathbb{R}^{n \times q}$. Then, $\bar{B}=\left(X^{\top} X\right)^{-1} X^{\top} Y$ is sufficient for $B$ and its distribution is $\bar{B} \sim \mathrm{N}_{p, q}\left(B,\left(X^{\top} X\right)^{-1}, \Sigma\right)$. Thus, multivariate linear regression reduces to estimation of $B$ based on $\bar{B}$. If $\hat{B}_{1}$ dominates $\hat{B}_{2}$ under the matrix quadratic loss $L(B, \hat{B})=(\hat{B}-B)(\hat{B}-B)^{\top}$, then $\hat{B}_{1}$ provides better estimation of mean response $B^{\top} x$ for any value of the explanatory variables $x$ : $E\left\{\left\|\left(\hat{B}_{1}-B\right)^{\top} x\right\|^{2}\right\} \leq E\left\{\left\|\left(\hat{B}_{2}-B\right)^{\top} x\right\|^{2}\right\}$.

Whereas we adopt the matrix quadratic loss in this study, most existing studies on estimation of a normal mean matrix used the Frobenius loss:

$$
l(M, \hat{M})=\|\hat{M}-M\|_{\mathrm{F}}^{2}=\sum_{a=1}^{n} \sum_{i=1}^{p}\left(\hat{M}_{a i}-M_{a i}\right)^{2},
$$

which is the trace of the matrix quadratic loss: $l(M, \hat{M})=\operatorname{tr} L(M, \hat{M})$. When $n-p-1>0$, Efron and Morris [4] proposed an estimator

$$
\hat{M}_{\mathrm{EM}}=X\left(I_{p}-(n-p-1)\left(X^{\top} X\right)^{-1}\right),
$$

which is viewed as a matrix version of the James-Stein estimator. They showed that $\hat{M}_{\mathrm{EM}}$ is minimax and dominates the maximum likelihood estimator $\hat{M}=X$ under the Frobenius loss. Note that $\hat{M}_{\mathrm{EM}}$ does not change the singular vectors but shrinks the singular values of $X$ towards zero [17]. Motivated from this property, Matsuda and Komaki [14] developed a singular value shrinkage prior

$$
\pi_{\mathrm{SVS}}(M)=\operatorname{det}\left(M^{\top} M\right)^{-(n-p-1) / 2},
$$

which is viewed as a matrix version of Stein's prior for a normal mean vector [17]. They proved that this prior is superharmonic and thus the generalized Bayes estimator with respect to $\pi_{\mathrm{SVS}}$ is minimax and dominates the maximum likelihood estimator under the Frobenius loss. Both the Efron-Morris estimator and the generalized Bayes estimator with respect to $\pi_{\mathrm{SvS}}$ have large risk reduction when $M$ is close to low rank, because they shrink the singular values separately.

In this study, we investigate shrinkage estimation of a normal mean matrix under the matrix quadratic loss. First, we derive an unbiased estimate of matrix quadratic risk for general estimators and prove that the Efron-Morris estimator (11) is minimax. Next, we introduce a notion of matrix superharmonicity for matrix-variate functions and show that it has analogous properties with usual superharmonic functions [9], which may be of independent interest. Then, we show that the generalized Bayes estimator with respect to a matrix superharmonic prior is minimax. For a normal mean vector $(p=1)$, it reduces to the result by Stein [17] on quadratic loss. We also provide a class of matrix superharmonic priors, which includes the singular value shrinkage prior (2). Finally, we give several numerical results, which demonstrate that matrix superharmonic priors work well for low rank matrices. 


\section{Estimation under matrix quadratic loss}

In this section, we provide preliminary results on estimation of a normal mean matrix under the matrix quadratic loss.

\subsection{Definition of minimaxity}

Since the matrix quadratic loss is only partially ordered, the definition of minimaxity under this loss is not straightforward. In this paper, we adopt the following definition.

Definition 2.1. An estimator $\hat{M}$ is said to be minimax under the matrix quadratic loss if

$$
\sup _{M} c^{\top} R(M, \hat{M}) c=\inf _{\hat{M}^{\prime}} \sup _{M} c^{\top} R\left(M, \hat{M}^{\prime}\right) c
$$

for every $c \in \mathbb{R}^{p}$.

Namely, we define $\hat{M}$ to be minimax under the matrix quadratic loss if $\hat{M} c$ is a minimax estimator of $M c$ under quadratic loss for every $c$, because $c^{\top} R(M, \hat{M}) c=\mathrm{E}_{M}\left[\|\hat{M} c-M c\|^{2}\right]$. In particular, since $X c$ is a minimax estimator of $M c$ for every $c$, the maximum likelihood estimator $\hat{M}=X$ is minimax under the matrix quadratic loss with constant risk $R(M, \hat{M})=$ $n I_{p}$. Thus, any estimator that dominates the maximum likelihood estimator is also minimax.

\subsection{Unbiased estimate of risk}

Here, we derive an unbiased estimate of the matrix quadratic risk. Our derivation is based on Stein's lemma for matrix-variate normal distributions expressed by a matrix version of divergence. Note that a locally integrable function $g: \mathbb{R}^{n \times p} \rightarrow \mathbb{R}^{n \times p}$ is said to be weakly differentiable [6] if there exist locally integrable functions $\partial_{a i} h$ for $a=1, \ldots, n$ and $i=1, \ldots, p$ such that

$$
\int h(X) \frac{\partial}{\partial X_{a i}} \phi(X) \mathrm{d} X=-\int \partial_{a i} h(X) \phi(X) \mathrm{d} X
$$

holds for any infinitely differentiable function $\phi: \mathbb{R}^{n \times p} \rightarrow \mathbb{R}$ with compact support.

Definition 2.2. For a function $g: \mathbb{R}^{n \times p} \rightarrow \mathbb{R}^{n \times p}$, its matrix divergence $\widetilde{\text { div }} g: \mathbb{R}^{n \times p} \rightarrow \mathbb{R}^{p \times p}$ is defined as

$$
(\widetilde{\operatorname{div}} g(X))_{i j}=\sum_{a=1}^{n} \frac{\partial}{\partial X_{a i}} g_{a j}(X) .
$$

Lemma 2.3. Let $X \sim \mathrm{N}_{n, p}\left(M, I_{n}, I_{p}\right)$ and $g: \mathbb{R}^{n \times p} \rightarrow \mathbb{R}^{n \times p}$ be a weakly differentiable function. Then,

$$
\mathrm{E}_{M}\left[(X-M)^{\top} g(X)\right]=\mathrm{E}_{M}[\widetilde{\operatorname{div}} g(X)] .
$$

Proof. By applying Stein's lemma [6],

$$
\begin{aligned}
\mathrm{E}_{M}\left[(X-M)^{\top} g(X)\right]_{i j} & =\mathrm{E}_{M}\left[\sum_{a}\left(X_{a i}-M_{a i}\right) g_{a j}(X)\right] \\
& =\mathrm{E}_{M}\left[\sum_{a} \frac{\partial}{\partial X_{a i}} g_{a j}(X)\right] \\
& =\mathrm{E}_{M}\left[(\widetilde{\operatorname{div}} g(X))_{i j}\right] .
\end{aligned}
$$


Theorem 2.4. The matrix quadratic risk of an estimator $\hat{M}=X+g(X)$ with a weakly differentiable function $g$ is given by

$$
R(M, \hat{M})=n I_{p}+\mathrm{E}_{M}\left[\widetilde{\operatorname{div}} g(X)+(\widetilde{\operatorname{div}} g(X))^{\top}+g(X)^{\top} g(X)\right] .
$$

Proof. By using Lemma 2.3 ,

$$
\begin{aligned}
& R(M, \hat{M}) \\
= & \mathrm{E}_{M}\left[(X+g(X)-M)^{\top}(X+g(X)-M)\right] \\
= & \mathrm{E}_{M}\left[(X-M)^{\top}(X-M)+(X-M)^{\top} g(X)+g(X)^{\top}(X-M)+g(X)^{\top} g(X)\right] \\
= & n I_{p}+\mathrm{E}_{M}\left[\widetilde{\operatorname{div}} g(X)+(\widetilde{\operatorname{div}} g(X))^{\top}+g(X)^{\top} g(X)\right] .
\end{aligned}
$$

\subsection{Minimaxity of Efron-Morris estimator}

By using Theorem 2.4, we show that the Efron-Morris estimator (1) is minimax under the matrix quadratic loss.

Theorem 2.5. When $n-p-1>0$, the Efron-Morris estimator $\hat{M}_{\mathrm{EM}}$ in (1) is minimax under the matrix quadratic loss.

Proof. Let $g(X)=-(n-p-1) X\left(X^{\top} X\right)^{-1}$ so that $\hat{M}_{\mathrm{EM}}=X+g(X)$.

To calculate $\widetilde{\operatorname{div}} g(X)$, we use the formula [13]

$$
\frac{\partial}{\partial X_{a i}}\left(X^{\top} X\right)^{-1}=-\left(X^{\top} X\right)^{-1}\left(X^{\top} E_{a i}+E_{a i}^{\top} X\right)\left(X^{\top} X\right)^{-1}
$$

where $E_{a i} \in \mathbb{R}^{n \times p}$ is the matrix unit with 1 in the $(a, i)$-th entry and 0s elsewhere. Then,

$$
\begin{aligned}
& \frac{\partial}{\partial X_{a i}}\left(X\left(X^{\top} X\right)^{-1}\right)_{a j} \\
= & \frac{\partial}{\partial X_{a i}} \sum_{k} X_{a k}\left(\left(X^{\top} X\right)^{-1}\right)_{k j} \\
= & \left(\left(X^{\top} X\right)^{-1}\right)_{i j}-\sum_{k, l, m} X_{a k}\left(\left(X^{\top} X\right)^{-1}\right)_{k l}\left(X^{\top} E_{a i}+E_{a i}^{\top} X\right)_{l m}\left(\left(X^{\top} X\right)^{-1}\right)_{m j} \\
= & \left(\left(X^{\top} X\right)^{-1}\right)_{i j}-\sum_{k, l, m} X_{a k}\left(\left(X^{\top} X\right)^{-1}\right)_{k l}\left(\delta_{i m} X_{a l}+\delta_{i l} X_{a m}\right)\left(\left(X^{\top} X\right)^{-1}\right)_{m j},
\end{aligned}
$$

where $\delta_{i j}$ is the Kronecker delta. Thus,

$$
\begin{aligned}
& \sum_{a} \frac{\partial}{\partial X_{a i}}\left(X\left(X^{\top} X\right)^{-1}\right)_{a j} \\
= & n\left(\left(X^{\top} X\right)^{-1}\right)_{i j}-\sum_{k, l, m}\left(\left(X^{\top} X\right)^{-1}\right)_{k l}\left(\delta_{i m}\left(X^{\top} X\right)_{k l}+\delta_{i l}\left(X^{\top} X\right)_{k m}\right)\left(\left(X^{\top} X\right)^{-1}\right)_{m j} \\
= & (n-p-1)\left(\left(X^{\top} X\right)^{-1}\right)_{i j} .
\end{aligned}
$$


Therefore,

$$
\widetilde{\operatorname{div}} g(X)=-(n-p-1)^{2}\left(X^{\top} X\right)^{-1}
$$

Also,

$$
g(X)^{\top} g(X)=(n-p-1)^{2}\left(X^{\top} X\right)^{-1}
$$

Therefore, from Theorem 2.4 .

$$
R\left(M, \hat{M}_{\mathrm{EM}}\right)=n I_{p}-(n-p-1)^{2} \mathrm{E}_{M}\left[\left(X^{\top} X\right)^{-1}\right] \preceq n I_{p},
$$

which means that $\hat{M}_{\mathrm{EM}}$ is minimax.

Corollary 2.6. The matrix quadratic risk of the Efron-Morris estimator at $M=O$ is

$$
R\left(O, \hat{M}_{\mathrm{EM}}\right)=(p+1) I_{p} .
$$

Proof. When $M=O$, the matrix $\left(X^{\top} X\right)^{-1}$ follows the inverse Wishart distribution $W^{-1}\left(n, I_{p}\right)$. Thus, from the formula for the mean of the inverse Wishart distribution [8],

$$
\mathrm{E}_{M=O}\left[\left(X^{\top} X\right)^{-1}\right]=(n-p-1)^{-1} I_{p}
$$

Therefore, from (3),

$$
R\left(O, \hat{M}_{\mathrm{EM}}\right)=n I_{p}-(n-p-1)^{2} \mathrm{E}_{M=O}\left[\left(X^{\top} X\right)^{-1}\right]=(p+1) I_{p} .
$$

We will study the matrix quadratic risk of the Efron-Morris estimator numerically in Section 5 .

Remark 2.7. Matsuda and Komaki [15] developed an empirical Bayes method for matrix completion based on the Efron-Morris estimator. It is an interesting future problem to investigate its performance in terms of the matrix quadratic loss.

\section{Matrix superharmonicity}

In this section, we introduce a notion of matrix superharmonicity for matrix-variate functions, which will be used to derive Bayes minimax estimators in the next section.

First, we review the definition of a superharmonic function [9, Definition 3.3.3]. Let $S_{x, r}=$ $\left\{x+r e \mid e \in \mathbb{R}^{n},\|e\|=1\right\} \subset \mathbb{R}^{n}$ be the sphere with center $x$ and radius $r>0$. For a function $f: \mathbb{R}^{n} \rightarrow \mathbb{R} \cup\{\infty\}$, let

$$
L(f: x, r)=\frac{1}{\Omega_{n} r^{n-1}} \int_{S_{x, r}} f(z) \mathrm{d} s(z)=\frac{1}{\Omega_{n}} \int_{S_{0,1}} f(x+r e) \mathrm{d} s(e)
$$

be the average value of $f$ on $S_{x, r}$, where d $s$ denotes the surface area element and $\Omega_{n}$ is the surface area of the unit sphere $S_{0,1}$ in $\mathbb{R}^{n}$. Then, $f$ is said to be superharmonic if it satisfies the following: 
1. $f$ is lower semicontinuous;

2. $f \not \equiv \infty$;

3. $L(f: x, r) \leq f(x)$ for every $x \in \mathbb{R}^{n}$ and $r>0$.

Now, we introduce matrix superharmonicity. For a matrix-variate function $f: \mathbb{R}^{n \times p} \rightarrow \mathbb{R} \cup$ $\{\infty\}$, we can define its superharmonicity as the superharmonicity of $f \circ \mathrm{vec}^{-1}: \mathbb{R}^{n p} \rightarrow \mathbb{R} \cup\{\infty\}$, where vec : $\mathbb{R}^{n \times p} \rightarrow \mathbb{R}^{n p}$ is the vectorization operator [8]. However, such a definition does not take into account the matrix structure. We propose a stronger version of superharmonicity for matrix-variate functions, which we refer to as matrix superharmonicity. For $X \in \mathbb{R}^{n \times p}$ and $\rho \in \mathbb{R}^{p}$, let $S_{X, \rho}=\left\{X+e \rho^{\top} \mid e \in \mathbb{R}^{n},\|e\|=1\right\}$ and

$$
L(f: X, \rho)=\frac{1}{\Omega_{n}} \int_{S_{0,1}} f\left(X+e \rho^{\top}\right) \mathrm{d} s(e)
$$

be the average value of $f$ on $S_{X, \rho}$. Note that we take average over only rank one perturbations around $X$ here.

Definition 3.1. A matrix-variate function $f: \mathbb{R}^{n \times p} \rightarrow \mathbb{R} \cup\{\infty\}$ is said to be matrix superharmonic if it satisfies the following:

1. $f$ is lower semicontinuous;

2. $f \not \equiv \infty$;

3. $L(f: X, \rho) \leq f(X)$ for every $X \in \mathbb{R}^{n \times p}$ and $\rho \in \mathbb{R}^{p}$.

We will provide examples of matrix superharmonic functions in Sections 4.3 and 4.4.

Proposition 3.2. If a function $f: \mathbb{R}^{n \times p} \rightarrow \mathbb{R} \cup\{\infty\}$ is matrix superharmonic, then $f \circ \mathrm{vec}^{-1}$ is superharmonic.

Proof. For every $X \in \mathbb{R}^{n \times p}$ and $r>0$,

$$
L\left(f \circ \operatorname{vec}^{-1}: \operatorname{vec}(X), r\right)=\frac{1}{\Omega_{p} r^{p-1}} \int_{S_{0, r}} L(f: X, \rho) \mathrm{d} s(\rho) .
$$

Since $L(f: X, \rho) \leq f(X)$ for every $\rho$ from the matrix superharmonicity of $f$,

$$
L\left(f \circ \operatorname{vec}^{-1}: \operatorname{vec}(X), r\right) \leq f(X) .
$$

The converse of Proposition 3.2 does not hold when $p \geq 2$. One counterexample is $f(X)=$ $\|X\|_{\mathrm{F}}^{2-n p}$ as we will show in Proposition 4.15.

Remark 3.3. When $n=1, S_{X, \rho}=\{X-\rho, X+\rho\}$ and thus the third condition of matrix superharmonicity reduces to midpoint convexity:

$$
L(f: X, \rho)=\frac{f(X+\rho)+f(X-\rho)}{2} \leq f(X),
$$

which is equivalent to usual convexity for a lower semicontinuous function $f$ on $\mathbb{R}^{p}$. On the other hand, the definition of midpoint convexity is not unique for functions defined on discrete space [16]. It may be interesting to investigate discrete analogue of matrix superharmonicity and its applications. 
We provide a characterization of matrix superharmonicity for $C^{2}$ functions. Recall that a $C^{2}$ function $f: \mathbb{R}^{n} \rightarrow \mathbb{R}$ is superharmonic if and only if its Laplacian is nonpositive:

$$
\Delta f(x)=\sum_{a=1}^{n} \frac{\partial^{2}}{\partial x_{a}^{2}} f(x) \leq 0
$$

for every $x$ [9, Lemma 3.3.4]. This property is extended to matrix superharmonic functions by using a matrix version of the Laplacian.

Definition 3.4. For a $C^{2}$ function $f: \mathbb{R}^{n \times p} \rightarrow \mathbb{R}$, its matrix Laplacian $\widetilde{\Delta} f: \mathbb{R}^{n \times p} \rightarrow \mathbb{R}^{p \times p}$ is defined as

$$
(\widetilde{\Delta} f(X))_{i j}=\sum_{a=1}^{n} \frac{\partial^{2}}{\partial X_{a i} \partial X_{a j}} f(X)
$$

Theorem 3.5. A $C^{2}$ function $f: \mathbb{R}^{n \times p} \rightarrow \mathbb{R}$ is matrix superharmonic if and only if its matrix Laplacian is negative semidefinite $\widetilde{\Delta} f(X) \preceq O$ for every $X$.

Proof. Assume that $f$ is matrix superharmonic. Let $e \in \mathbb{R}^{n}$ be a unit vector. From Taylor's theorem,

$$
\begin{aligned}
& f\left(X+e \rho^{\top}\right) \\
= & f(X)+\sum_{a, i} \frac{\partial f}{\partial X_{a i}}(X) e_{a} \rho_{i}+\frac{1}{2} \sum_{a, i, b, j} \frac{\partial^{2} f}{\partial X_{a i} \partial X_{b j}}(X) e_{a} \rho_{i} e_{b} \rho_{j}+o\left(\|\rho\|^{2}\right) .
\end{aligned}
$$

Thus,

$$
\begin{aligned}
L(f: X, \rho) & =\frac{1}{\Omega_{n}} \int_{S_{0,1}} f\left(X+e \rho^{\top}\right) \mathrm{d} s(e) \\
& =f(X)+\frac{1}{2 n} \sum_{a, i, j} \frac{\partial^{2} f}{\partial X_{a i} \partial X_{a j}}(X) \rho_{i} \rho_{j}+o\left(\|\rho\|^{2}\right) \\
& =f(X)+\frac{1}{2 n} \rho^{\top} \widetilde{\Delta} f(X) \rho+o\left(\|\rho\|^{2}\right),
\end{aligned}
$$

where we used

$$
\frac{1}{\Omega_{n}} \int_{S_{0,1}} e_{a} \mathrm{~d} s(e)=0, \quad \frac{1}{\Omega_{n}} \int_{S_{0,1}} e_{a} e_{b} \mathrm{~d} s(e)=\frac{1}{n} \delta_{a b} .
$$

On the other hand, $L(f: X, \rho) \leq f(X)$ from the matrix superharmonicity of $f$. Therefore, we must have

$$
\rho^{\top} \widetilde{\Delta} f(X) \rho \leq 0
$$

for every $\rho$. Hence, $\widetilde{\Delta} f(X) \preceq O$ for every $X$.

Conversely, assume that $\widetilde{\Delta} f(X) \preceq O$ for every $X$. For arbitrary $X$ and $\rho$, let $\bar{f}(e)=$ $f\left(X+e \rho^{\top}\right)$. Then,

$$
\Delta \bar{f}(e)=\sum_{a} \frac{\partial^{2} \bar{f}}{\partial e_{a}^{2}}=\sum_{a, i} \frac{\partial}{\partial e_{a}}\left(\frac{\partial f}{\partial X_{a i}} \rho_{i}\right)=\sum_{a, i, j} \rho_{i} \frac{\partial^{2} f}{\partial X_{a i} \partial X_{a j}} \rho_{j}=\rho^{\top} \widetilde{\Delta} f\left(X+e \rho^{\top}\right) \rho \leq 0 .
$$


Let $C_{\eta}=\left\{e \in \mathbb{R}^{n} \mid \eta<\|e\|<1\right\}$ and $\bar{g}(e)=\|e\|^{2-n}-1$. Then, $\bar{g} \geq 0$ and $\Delta \bar{g}=0$ on $C_{\eta}$. Therefore, from Green's theorem,

$$
\int_{\partial C_{\eta}}\left(\bar{f} D_{n} \bar{g}-\bar{g} D_{n} \bar{f}\right) \mathrm{d} s(e)=\int_{C_{\eta}}(\bar{f} \Delta \bar{g}-\bar{g} \Delta \bar{f}) \mathrm{d} e \geq 0,
$$

where $\partial C_{\eta}=S_{0, \eta} \cup S_{0,1}$ is the boundary of $C_{\eta}$ and $D_{n} \bar{f}$ is the directional derivative of $\bar{f}$ in the direction of the outer normal unit vector. On the other hand, since $D_{n} \bar{g}=-(2-n) \eta^{1-n}$ on $S_{0, \eta}$ and $D_{n} \bar{g}=2-n$ on $S_{0,1}$,

$$
\begin{aligned}
\int_{\partial C_{\eta}} \bar{f} D_{n} \bar{g} \mathrm{~d} s(e) & =-(2-n) \eta^{1-n} \int_{S_{0, \eta}} \bar{f} \mathrm{~d} s(e)+(2-n) \int_{S_{0,1}} \bar{f} \mathrm{~d} s(e) \\
& =-(2-n) \Omega_{n} L(f: X, \eta \rho)+(2-n) \Omega_{n} L(f: X, \rho) .
\end{aligned}
$$

Also, since $\bar{g}=\eta^{2-n}-1$ on $S_{0, \eta}$ and $\bar{g}=0$ on $S_{0,1}$,

$$
\int_{\partial C_{\eta}} \bar{g} D_{n} \bar{f} \mathrm{~d} s(e)=\left(\eta^{2-n}-1\right) \int_{S_{0, \eta}} D_{n} \bar{f} \mathrm{~d} s(e)
$$

which is $O(\eta)$ as $\eta \rightarrow 0$. Therefore,

$$
L(f: X, \rho) \leq L(f: X, \eta \rho)+O(\eta) .
$$

By taking $\eta \rightarrow 0$, we obtain $L(f: X, \rho) \leq f(X)$. Since $X$ and $\rho$ are arbitrary, $f$ is matrix superharmonic.

The limit of an increasing sequence of superharmonic functions is also superharmonic [9, Theorem 3.4.8]. Matrix superharmonic functions have a similar property.

Lemma 3.6. Let $f_{1} \leq f_{2} \leq \ldots$ be an increasing sequence of matrix superharmonic functions and assume that $f=\lim _{k \rightarrow \infty} f_{k} \not \equiv \infty$. Then, $f$ is also matrix superharmonic.

Proof. Since each $f_{k}$ is lower semicontinuous, their supremum $f$ is also lower semicontinuous. Also, from Lemma 3.2.10 of [9] ,

$$
\int_{S_{0,1}} f\left(X+e \rho^{\top}\right) \mathrm{d} s(e)=\lim _{k \rightarrow \infty} \int_{S_{0,1}} f_{k}\left(X+e \rho^{\top}\right) \mathrm{d} s(e)
$$

Therefore,

$$
L(f: X, \rho)=\lim _{k \rightarrow \infty} L\left(f_{k}: X, \rho\right) \leq \lim _{k \rightarrow \infty} f_{k}(X)=f(X),
$$

where we used the matrix superharmonicity of each $f_{k}$.

\section{Bayes estimation with matrix superharmonic prior}

In this section, we investigate Bayes shrinkage estimation under the matrix quadratic loss. 


\subsection{Uniqueness of Bayes estimator}

Let

$$
m_{\pi}(X)=\int p(X \mid M) \pi(M) \mathrm{d} M
$$

be the marginal distribution of $X \sim \mathrm{N}_{n, p}\left(M, I_{n}, I_{p}\right)$ with prior $\pi(M)$.

Similarly to the Frobenius loss, the (generalized) Bayes estimator under the matrix quadratic loss is uniquely given by the posterior mean, even though the matrix quadratic loss is only partially ordered.

Definition 4.1. For a function $f: \mathbb{R}^{n \times p} \rightarrow \mathbb{R}$, its matrix gradient $\widetilde{\nabla} f: \mathbb{R}^{n \times p} \rightarrow \mathbb{R}^{n \times p}$ is defined as

$$
(\widetilde{\nabla} f(X))_{a i}=\frac{\partial}{\partial X_{a i}} f(X) .
$$

Lemma 4.2. If $m_{\pi}(X)<\infty$ for every $X$, then the (generalized) Bayes estimator of $M$ with respect to a prior $\pi(M)$ under the matrix quadratic loss is uniquely given by the posterior mean:

$$
\hat{M}^{\pi}(X)=\mathrm{E}_{\pi}[M \mid X]=\frac{\int M p(X \mid M) \pi(M) \mathrm{d} M}{\int p(X \mid M) \pi(M) \mathrm{d} M}=X+\widetilde{\nabla} \log m_{\pi}(X) .
$$

Proof. For each estimator $\hat{M}=\hat{M}(X)$, its posterior risk is decomposed as

$$
\begin{aligned}
& \mathrm{E}_{\pi}\left[(\hat{M}(X)-M)^{\top}(\hat{M}(X)-M) \mid X\right] \\
= & \mathrm{E}_{\pi}\left[\left(\mathrm{E}_{\pi}[M \mid X]-M\right)^{\top}\left(\mathrm{E}_{\pi}[M \mid X]-M\right)\right]+D^{\top} D,
\end{aligned}
$$

where $D=\hat{M}(X)-\mathrm{E}_{\pi}[M \mid X]$. Thus, the posterior risk is uniquely minimized under the Lowner order by taking $D=O$, which means that $\hat{M}(X)=\mathrm{E}_{\pi}[M \mid X]$.

\subsection{Sufficient condition for minimaxity}

Now, we provide a sufficient condition for minimaxity of (generalized) Bayes estimators under the matrix quadratic loss.

Theorem 4.3. If $\sqrt{m_{\pi}(X)}$ is matrix superharmonic, then the generalized Bayes estimator $\hat{M}^{\pi}(X)=X+\widetilde{\nabla} \log m_{\pi}(X)$ with respect to $\pi(M)$ is minimax under the matrix quadratic loss.

Proof. From Theorem 2.4, the matrix quadratic risk of the generalized Bayes estimator $\hat{M}^{\pi}$ is given by

$$
\begin{aligned}
R\left(M, \hat{M}^{\pi}\right) & =n I_{p}+\mathrm{E}_{M}\left[2 \widetilde{\Delta} \log m_{\pi}(X)+\left(\widetilde{\nabla} \log m_{\pi}(X)\right)^{\top}\left(\widetilde{\nabla} \log m_{\pi}(X)\right)\right] \\
& =n I_{p}+4 \mathrm{E}_{M}\left[\frac{\widetilde{\Delta} \sqrt{m_{\pi}(X)}}{\sqrt{m_{\pi}(X)}}\right] .
\end{aligned}
$$

Since $\sqrt{m_{\pi}(X)}$ is matrix superharmonic and $C^{2}$ by definition, $\widetilde{\Delta} \sqrt{m_{\pi}(X)} \preceq O$ for every $X$ from Theorem 3.5. Therefore, $R\left(M, \hat{M}^{\pi}\right) \preceq n I_{p}$ and thus $\hat{M}^{\pi}$ is minimax. 

itself.

We also provide another sufficient condition based on the matrix superharmonicity of prior

Lemma 4.4. If $\pi(M)$ is matrix superharmonic and $m_{\pi}(X)<\infty$ for every $X$, then $m_{\pi}(X)$ is also matrix superharmonic.

Proof. Let $\phi(Z)$ be the probability density of $Z \sim \mathrm{N}_{n, p}\left(O, I_{n}, I_{p}\right)$. Then,

$$
m_{\pi}(Z)=\int \phi(Z-M) \pi(M) \mathrm{d} M=\int \phi(A) \pi(Z-A) \mathrm{d} A .
$$

Thus, for every $X \in \mathbb{R}^{n \times p}$ and $\rho \in \mathbb{R}^{p}$,

$$
\begin{aligned}
L\left(m_{\pi}: X, \rho\right) & =\frac{1}{\Omega_{n}} \int_{S_{0,1}}\left\{\int \phi(A) \pi\left(X+e \rho^{\top}-A\right) \mathrm{d} A\right\} \mathrm{d} s(e) \\
& =\frac{1}{\Omega_{n}} \int \phi(A)\left\{\int_{S_{0,1}} \pi\left(X+e \rho^{\top}-A\right) \mathrm{d} s(e)\right\} \mathrm{d} A \\
& =\int \phi(A) L(\pi: X-A, \rho) \mathrm{d} A \\
& \leq \int \phi(A) \pi(X-A) \mathrm{d} A \\
& =m_{\pi}(X),
\end{aligned}
$$

where the second equation follows from Fubini's theorem and the inequality follows from the matrix superharmonicity of $\pi$. Therefore, $m_{\pi}$ is matrix superharmonic.

Lemma 4.5. If $f: \mathbb{R}^{n \times p} \rightarrow \mathbb{R}$ is matrix superharmonic and $\phi: \mathbb{R} \rightarrow \mathbb{R}$ is monotone increasing and concave in the range of $f$, then $\phi \circ f$ is also matrix superharmonic. In particular, if $f$ is matrix superharmonic and non-negative, then $\sqrt{f}$ is also matrix superharmonic.

Proof. For every $X \in \mathbb{R}^{n \times p}$ and $\rho \in \mathbb{R}^{p}$,

$$
\begin{aligned}
L(\phi \circ f: X, \rho) & =\frac{1}{\Omega_{n}} \int_{S_{0,1}} \phi\left(f\left(X+e \rho^{\top}\right)\right) \mathrm{d} s(e) \\
& \leq \phi\left(\frac{1}{\Omega_{n}} \int_{S_{0,1}} f\left(X+e \rho^{\top}\right) \mathrm{d} s(e)\right) \\
& =\phi(L(f: X, \rho)) \\
& \leq \phi(f(X)),
\end{aligned}
$$

where the first inequality follows from Jensen's inequality and the second inequality follows from the matrix superharmonicity of $f$ and the monotonicity of $\phi$. Therefore, $\phi \circ f$ is matrix superharmonic.

Theorem 4.6. Let $\pi(M)$ be a superharmonic prior with $m_{\pi}(X)<\infty$ for every $X$. Then, the generalized Bayes estimator $\hat{M}^{\pi}(X)=X+\widetilde{\nabla} \log m_{\pi}(X)$ with respect to $\pi(M)$ is minimax under the matrix quadratic loss.

Proof. From Lemma 4.4 and Lemma 4.5, $\sqrt{m_{\pi}(X)}$ is matrix superharmonic. Therefore, from Theorem 4.3, $\hat{M}^{\pi}$ is minimax. 
When $p=1$, Theorem 4.3 and Theorem 4.6 reduce to the classical results by Stein [17] on minimax Bayes estimators of a normal mean vector.

Remark 4.7. Whereas matrix superharmonic priors provide minimax Bayes estimators of any linear combination $M c$ simultaneously, there may be cases where we are only interested in linear combination with nonnegative coefficients $c \in \mathbb{R}_{+}^{p}$. In such cases, only the copositivity [2] of $-\widetilde{\Delta} m_{\pi}(X)$ suffices. Thus, it may be interesting to develop another version of matrix superharmonicity based on copositivity.

Remark 4.8. Recently, superharmonic priors have been found to give minimax predictive densities under the Kullback-Leibler loss [11, 7]. It is an interesting future problem to investigate properties of matrix superharmonic priors in predictive density estimation under some analogue of matrix quadratic loss.

\subsection{Matrix superharmonic priors}

Here, we provide a class of matrix superharmonic priors, which includes the previously proposed generalization (2) of Stein's prior.

Let

$$
\pi_{\alpha, \beta}(M)=\operatorname{det}\left(M^{\top} M+\beta I_{p}\right)^{-(\alpha+n+p-1) / 2},
$$

where $-n-p+1 \leq \alpha \leq-2 p$ and $\beta \geq 0$. When $\alpha=-2 p$ and $\beta=0, \pi_{\alpha, \beta}(M)$ coincides with the singular value shrinkage prior (2). Note that $\pi_{\alpha, \beta}(M)$ with $\alpha>0$ and $\beta>0$ is the so-called matrix t-distribution with $\alpha$ degrees of freedom [8]. When $p=1, \pi_{\alpha, \beta}(M)$ reduces to the $n$-dimensional (improper) multivariate t-prior [5]

$$
\pi_{\alpha, \beta}(\mu)=\left(\|\mu\|^{2}+\beta\right)^{-(\alpha+n) / 2},
$$

and it is superharmonic when $-n \leq \alpha \leq-2$ and $\beta \geq 0$. This result is extended to general $p$ as follows.

Theorem 4.9. If $-n-p+1 \leq \alpha \leq-2 p$ and $\beta \geq 0$, then the prior $\pi_{\alpha, \beta}(M)$ in (5) is matrix superharmonic.

Proof. In the following, the subscripts $a, b, \ldots$ run from 1 to $n$ and the subscripts $i, j, \ldots$ run from 1 to $p$. We denote the $(i, j)$-th entry of $S^{-1}$ by $S^{i j}$ and the Kronecker delta by $\delta_{i j}$.

First, assume that $\beta>0$. Let $S=M^{\top} M+\beta I_{p} \succ O$. Since $S_{i j}=\sum_{a} M_{a i} M_{a j}+\beta \delta_{i j}$,

$$
\frac{\partial S_{k l}}{\partial M_{a i}}=\delta_{i k} M_{a l}+\delta_{i l} M_{a k}
$$

Also,

$$
\frac{\partial}{\partial S_{i j}} \operatorname{det} S=S^{i j} \operatorname{det} S
$$

Thus,

$$
\frac{\partial}{\partial M_{a i}} \operatorname{det} S=\sum_{k, l} \frac{\partial S_{k l}}{\partial M_{a i}} \frac{\partial}{\partial S_{k l}} \operatorname{det} S=2 \sum_{k} M_{a k} S^{i k} \operatorname{det} S
$$


Therefore,

$$
\begin{aligned}
& \frac{\partial^{2}}{\partial M_{a i} \partial M_{a j}} \operatorname{det} S \\
= & 2\left(S^{i j}-\sum_{k, l} M_{a k} M_{a l} S^{i j} S^{k l}+\sum_{k, l} M_{a k} M_{a l} S^{i k} S^{j l}\right) \operatorname{det} S,
\end{aligned}
$$

where we used

$$
\frac{\partial S^{i k}}{\partial M_{a j}}=-\sum_{l, m} S^{i l} S^{k m} \frac{\partial S_{l m}}{\partial M_{a j}}=-\sum_{l} M_{a l} S^{i j} S^{k l}-\sum_{l} M_{a l} S^{i l} S^{j k}
$$

which is obtained by differentiating $\sum_{k} S_{j k} S^{i k}=\delta_{i j}$ and using (6).

Now,

$$
\begin{aligned}
\left(\widetilde{\Delta}(\operatorname{det} S)^{-(\alpha+n+p-1) / 2}\right)_{i j} & =\sum_{a} \frac{\partial^{2}}{\partial M_{a i} \partial M_{a j}}(\operatorname{det} S)^{-(\alpha+n+p-1) / 2} \\
& =\frac{\alpha+n+p-1}{2}(\operatorname{det} S)^{-(\alpha+n+p-1) / 2} \sum_{a}\left(A_{a i j}+B_{a i j}\right)
\end{aligned}
$$

where

$$
\begin{aligned}
A_{a i j} & =\frac{\alpha+n+p+1}{2}(\operatorname{det} S)^{-2}\left(\frac{\partial}{\partial M_{a i}} \operatorname{det} S\right)\left(\frac{\partial}{\partial M_{a j}} \operatorname{det} S\right), \\
B_{a i j} & =-(\operatorname{det} S)^{-1} \frac{\partial^{2}}{\partial M_{a i} \partial M_{a j}} \operatorname{det} S .
\end{aligned}
$$

By using (7) and $\sum_{a} M_{a k} M_{a l}=S_{k l}-\beta \delta_{k l}$,

$$
\begin{aligned}
\sum_{a} A_{a i j} & =2(\alpha+n+p+1) \sum_{a}\left(\sum_{k} M_{a k} S^{i k}\right)\left(\sum_{l} M_{a l} S^{j l}\right) \\
& =2(\alpha+n+p+1)\left(S^{i j}-\beta \sum_{k} S^{i k} S^{j k}\right) .
\end{aligned}
$$

On the other hand, from (8),

$$
\sum_{a} B_{a i j}=-2(n-p+1) S^{i j}-2 \beta S^{i j} \sum_{k} S^{k k}+2 \beta \sum_{k} S^{i k} S^{j k} .
$$

Hence,

$$
\sum_{a}\left(A_{a i j}+B_{a i j}\right)=2(\alpha+2 p) S^{i j}-2(\alpha+n+p) \beta \sum_{k} S^{i k} S^{j k}-2 \beta S^{i j} \sum_{k} S^{k k} .
$$

Substituting this expression into (9) gives

$$
\begin{aligned}
\widetilde{\Delta}(\operatorname{det} S)^{-(\alpha+n+p-1) / 2} & =\frac{\alpha+n+p-1}{2}(\operatorname{det} S)^{-(\alpha+n+p-1) / 2} \\
& \times\left(2(\alpha+2 p) S^{-1}-2(\alpha+n+p) \beta\left(S^{-1}\right)^{2}-2 \beta \operatorname{tr}\left(S^{-1}\right) S^{-1}\right),
\end{aligned}
$$


which is negative semidefinite from $-n-p+1 \leq \alpha \leq-2 p, \beta>0$ and $S^{-1} \succ O$. Therefore, by Theorem 3.5, $\pi_{\alpha, \beta}(M)=(\operatorname{det} S)^{-(\alpha+n+p-1) / 2}$ is matrix superharmonic.

Next, assume that $\beta=0$. Let

$$
\pi^{(k)}(M)=\operatorname{det}\left(M^{\top} M+k^{-1} I_{p}\right)^{-(\alpha+n+p-1) / 2}, \quad k=1,2, \ldots
$$

Then, each $\pi^{(k)}$ is matrix superharmonic from the above discussion. Also, $\pi^{(1)} \leq \pi^{(2)} \leq \cdots$ and $\lim _{k \rightarrow \infty} \pi^{(k)}(M)=\pi_{\alpha, \beta}(M)$ for every $M$. Therefore, from Lemma 3.6, $\pi_{\alpha, \beta}(M)$ is also matrix superharmonic.

Proposition 4.10. If $-n-p+1 \leq \alpha \leq-2 p$ and $\beta \geq 0$, then the marginal density $m_{\pi}(X)$ in (4) with $\pi=\pi_{\alpha, \beta}$ in (5) is finite for every $X$.

Proof. We use the fact that $m_{\pi}(X)$ in (44) is interpreted as the expected value of $\pi(M)$ with respect to $M \sim \mathrm{N}_{n, p}\left(X, I_{n}, I_{p}\right)$.

When $\beta>0$, since $\pi_{\alpha, \beta}(M) \leq \pi_{\alpha, \beta}(O)=\beta^{-p(\alpha+n+p-1) / 2}$,

$$
m_{\pi}(X) \leq \beta^{-p(\alpha+n+p-1) / 2}
$$

for every $X$.

When $\beta=0$,

$$
m_{\pi}(X)=\mathrm{E}\left[(\operatorname{det} S)^{-(\alpha+n+p-1) / 2}\right]
$$

where $S=M^{\top} M$ has a noncentral Wishart distribution $S \sim W_{p}\left(n, I_{p}, X^{\top} X\right)$ from Theorem 3.5.1 in [8]. Therefore, by using Theorem 3.5.6 of [8],

$$
m_{\pi}(X)=C \operatorname{etr}\left(-\frac{1}{2} X^{\top} X\right){ }_{1} F_{1}\left(-\frac{\alpha+p-1}{2} ; \frac{n}{2} ; \frac{1}{2} X^{\top} X\right)
$$

where $C=2^{-p(\alpha+n+p-1) / 2} \Gamma_{p}(-(\alpha+p-1) / 2) / \Gamma_{p}(n / 2), \Gamma_{p}$ is the multivariate Gamma function and ${ }_{1} F_{1}$ is the hypergeometric function of a matrix argument [8]. Thus, $m_{\pi}(X)$ is finite for every $X$.

From Theorem 4.6, Theorem 4.9 and Proposition 4.10, we obtain the following.

Theorem 4.11. If $-n-p+1 \leq \alpha \leq-2 p$ and $\beta \geq 0$, then the generalized Bayes estimator with respect to the prior $\pi_{\alpha, \beta}(M)$ in (5) is minimax under the matrix quadratic loss.

By taking $\alpha=-2 p$ and $\beta=0$ in Theorem 4.9 and Theorem 4.11, we obtain the following result on the singular value shrinkage prior (2).

Corollary 4.12. When $n-p-1>0$, the singular value shrinkage prior $\pi_{\mathrm{SVS}}(M)$ in (2) is matrix superharmonic. Also, the generalized Bayes estimator with respect to $\pi_{\mathrm{SVS}}(M)$ is minimax under the matrix quadratic loss.

In particular, the matrix superharmonicity of $\pi_{\text {SVS }}$ is strongly concentrated on the space of low rank matrices, which has measure zero, in the same way as the Laplacian of Stein's prior $\pi(\mu)=\|\mu\|^{2-n}$ becomes a Dirac delta function at the origin. 
Corollary 4.13. If $M$ has full-rank, then $\widetilde{\Delta} \pi_{\mathrm{SVS}}(M)=O$.

Proof. Substituting $\alpha=-2 p$ and $\beta=0$ into (10) gives

$$
\widetilde{\Delta}(\operatorname{det} S)^{-(n-p-1) / 2}=-(n-p-1)(\operatorname{det} S)^{-(n-p-1) / 2}\left\{(n-p)\left(S^{-1}\right)^{2}+\operatorname{tr}\left(S^{-1}\right) S^{-1}\right\} \beta=O,
$$

where we used $\operatorname{det} S>0$ since $M$ has full-rank.

Remark 4.14. The Efron-Morris estimator (11) can be viewed as a pseudo-Bayes estimator $\hat{M}=X+\widetilde{\nabla} \log m(X)[\underline{6}]$ with the pseudo-marginal $m(X)=\pi_{\mathrm{SVS}}(X)=\operatorname{det}\left(X^{\top} X\right)^{-(n-p-1) / 2}$. Combining such a pseudo-Bayes interpretation with Theorem 4.3 and Theorem 4.9 with $\beta=0$, it follows that the estimator $\hat{M}=X\left(I_{p}-c\left(X^{\top} X\right)^{-1}\right)$ is minimax for $0 \leq c \leq 2(n-p-1)$.

\subsection{Stein-type priors}

We further investigate matrix superharmonicity of another types of shrinkage priors.

Since Stein's prior $\pi(\mu)=\|\mu\|^{2-n}$ for $\mu \in \mathbb{R}^{n}$ is superharmonic [17], the prior

$$
\pi_{\mathrm{S}}(M)=\|M\|_{\mathrm{F}}^{2-n p}=\|\operatorname{vec}(M)\|^{2-n p}
$$

is also superharmonic. More generally, the shrinkage prior $\pi(M)=\|M\|^{-c}$ with $c \geq 0$ is superharmonic if and only if $0 \leq c \leq n p-2[\underline{6}]$. However, the range of $c$ for matrix superharmoncity is narrower. In particular, Stein's prior $\pi_{\mathrm{S}}(M)$ in (11) is not matrix superharmonic.

Proposition 4.15. The prior $\pi(M)=\|M\|_{\mathrm{F}}^{-c}$ with $c \geq 0$ is matrix superharmonic if and only if $0 \leq c \leq n-2$.

Proof. First, assume that $0 \leq c \leq n-2$. Let $\pi_{\beta}(M)=\left(\|M\|_{\mathrm{F}}^{2}+\beta\right)^{-c / 2}$ with $\beta>0$. Then, $\pi_{\beta}(M)$ is $C^{2}$ and its matrix Laplacian is

$$
\widetilde{\Delta} \pi_{\beta}(M)=-c\left(\|M\|_{\mathrm{F}}^{2}+\beta\right)^{-c / 2-2}\left(n\left(\|M\|_{\mathrm{F}}^{2}+\beta\right) I_{p}-(c+2) M^{\top} M\right) \preceq O,
$$

where we used $n\left(\|M\|_{\mathrm{F}}^{2}+\beta\right) I_{p}-(c+2) M^{\top} M \succeq O$ from $n \geq c+2$ and $\|M\|_{\mathrm{F}}^{2} I_{p} \succeq M^{\top} M$. Therefore, from Theorem 3.5, $\pi_{\beta}(M)$ is matrix superharmonic for every $\beta>0$. Then, $\pi^{(k)}(M)=\left(\|M\|_{\mathrm{F}}^{2}+k^{-1}\right)^{-c / 2}$ is an increasing sequence of matrix superharmonic functions with $\lim _{k \rightarrow \infty} \pi^{(k)}(M)=\pi(M)$. Thus, from Lemma 3.6, $\pi(M)$ is also matrix superharmonic.

Next, assume that $c>n-2$. Consider $M$ and $\rho$ defined by

$$
M_{a i}=\left\{\begin{array}{ll}
1 & (i=1) \\
0 & (2 \leq i \leq p)
\end{array}, \quad \rho_{i}=\left\{\begin{array}{ll}
1 & (i=1) \\
0 & (2 \leq i \leq p)
\end{array} .\right.\right.
$$

Then,

$$
L(\pi: M, \rho)=\frac{1}{\Omega_{n}} \int_{S_{0,1}} \pi\left(M+e \rho^{\top}\right) \mathrm{d} s(e)=\frac{1}{\Omega_{n}} \int_{S_{0,1}} g(e) \mathrm{d} s(e),
$$

where the function $g: \mathbb{R}^{n} \rightarrow \mathbb{R}$ is given by $g(e)=\|\mathbf{1}+e\|^{-c}$ with the all-one vector $\mathbf{1}=$ $(1, \ldots, 1)^{\top} \in \mathbb{R}^{n}$. Since $c>n-2, \Delta g(e)=c(c-n+2)\|\mathbf{1}+e\|^{-c-2}>0$. Thus, by Green's theorem,

$$
\frac{1}{\Omega_{n}} \int_{S_{0,1}} g(e) \mathrm{d} s(e)>g(0)=\pi(M) .
$$

Hence, we have $L(\pi: M, \rho)>\pi(M)$. Therefore, $\pi(M)$ is not matrix superharmonic. 
Corollary 4.16. When $p \geq 2$, Stein's prior $\pi_{\mathrm{S}}(M)=\|M\|_{\mathrm{F}}^{2-n p}$ is not matrix superharmonic.

[1] showed that the column-wise shrinkage estimator of James-Stein type

$$
\hat{M}=X D, \quad D=\operatorname{diag}\left(d_{1}, \ldots, d_{p}\right), \quad d_{i}=1-\frac{c}{\sum_{a} X_{a i}^{2}}
$$

is minimax under the matrix quadratic loss when $0 \leq c \leq 2(n-2) / p$. Note that this estimator can be viewed as a pseudo-Bayes estimator $\hat{M}=X+\widetilde{\nabla} \log m(X)$ [6] with the pseudo-marginal $m(X)=\prod_{i}\left(\sum_{a} X_{a i}^{2}\right)^{-c / 2}$. Their result is understood from the viewpoint of matrix superharmonicity as follows.

Proposition 4.17. The prior $\pi(M)=\prod_{i}\left(\sum_{a} M_{a i}^{2}\right)^{-c / 2}$ with $c \geq 0$ is matrix superharmonic if and only if $0 \leq c \leq(n-2) / p$.

Proof. First, assume that $0 \leq c \leq(n-2) / p$. Let $\pi_{\beta}(M)=\prod_{i}\left(\sum_{a} M_{a i}^{2}+\beta\right)^{-c / 2}$ with $\beta>0$. Then, $\pi_{\beta}(M)$ is $C^{2}$ and its matrix Laplacian is

$$
\widetilde{\Delta} \pi_{\beta}(M)=c \pi_{\beta}(M)(c A-(n-2) B-n \beta C),
$$

where $A, B, C$ are $p \times p$ positive semidefinite matrices with entries

$$
\begin{gathered}
A_{i j}=\left(\sum_{a} M_{a i}^{2}+\beta\right)^{-1}\left(\sum_{a} M_{a j}^{2}+\beta\right)^{-1}\left(\sum_{a} M_{a i} M_{a j}\right) \\
B_{i j}=\delta_{i j} A_{i j}, \quad C_{i j}=\delta_{i j}\left(\sum_{a} M_{a i}^{2}+\beta\right)^{-2} .
\end{gathered}
$$

Let $S=B^{-1 / 2} A B^{-1 / 2} \succeq O$. Then, all diagonal entries of $S$ are one and thus $S \preceq(\operatorname{tr} S) I_{p}=p I_{p}$. Thus, $A=B^{1 / 2} S B^{1 / 2} \preceq p B$. Therefore, from $c \leq(n-2) / p$,

$$
\widetilde{\Delta} \pi_{\beta}(M) \preceq c(c p-n+2) \pi_{\beta}(M) B \preceq O .
$$

Hence, from Theorem 3.5, $\pi_{\beta}(M)$ is matrix superharmonic for every $\beta>0$. Then, $\pi^{(k)}(M)=$ $\prod_{i}\left(\sum_{a} M_{a i}^{2}+k^{-1}\right)^{-c / 2}$ is an increasing sequence of matrix superharmonic functions with $\lim _{k \rightarrow \infty} \pi^{(k)}(M)=\pi(M)$. Thus, from Lemma 3.6, $\pi(M)$ is also matrix superharmonic.

Next, assume that $c>(n-2) / p$. Suppose that all entries of $M$ and $\rho$ are one. Then,

$$
L(\pi: M, \rho)=\frac{1}{\Omega_{n}} \int_{S_{0,1}} \pi\left(M+e \rho^{\top}\right) \mathrm{d} s(e)=\frac{1}{\Omega_{n}} \int_{S_{0,1}} g(e) \mathrm{d} s(e),
$$

where the function $g: \mathbb{R}^{n} \rightarrow \mathbb{R}$ is given by $g(e)=\|\mathbf{1}+e\|^{-c p}$ with the all-one vector $\mathbf{1}=$ $(1, \ldots, 1)^{\top} \in \mathbb{R}^{n}$. Since $c>(n-2) / p, \Delta g(e)=c p(c p-n+2)\|\mathbf{1}+e\|^{-c p-2}>0$. Thus, by Green's theorem,

$$
\frac{1}{\Omega_{n}} \int_{S_{0,1}} g(e) \mathrm{d} s(e)>g(0)=\pi(M)
$$

Hence, we have $L(\pi: M, \rho)>\pi(M)$. Therefore, $\pi(M)$ is not matrix superharmonic. 


\section{$5 \quad$ Numerical results}

In this section, we present several numerical results on the matrix quadratic risk of shrinkage estimators. We denote the $i$-th singular value of $M$ by $\sigma_{i}$. Note that singular values are in descending order: $\sigma_{1} \geq \sigma_{2} \geq \cdots \geq \sigma_{p}$.

In the following, we focus on the eigenvalues $\lambda_{1} \geq \cdots \geq \lambda_{p}$ of the matrix quadratic risk $R(M, \hat{M})$ of several estimators. Since $R(M, \hat{M})=n I_{p}$ for the maximum likelihood estimator $\hat{M}=X$, an estimator is minimax if and only if $\lambda_{1} \leq n$ for every $M$.

First, we compare the generalized Bayes estimators with respect to the singular value shrinkage prior $\pi_{\mathrm{SVS}}(M)$ in (2) and Stein's prior $\pi_{\mathrm{S}}(M)$ in (11) for $n=5$ and $p=3$. We employed the numerical method of [14] to compute the generalized Bayes estimators.

Figure 1 plots the three eigenvalues $\lambda_{1} \geq \lambda_{2} \geq \lambda_{3}$ of the matrix quadratic risk with respect to $\sigma_{2}$ when $\sigma_{1}=10$ and $\sigma_{3}=0$. For $\pi_{\mathrm{SVS}}$, all eigenvalues do not exceed $n=5$, which indicates the minimaxity. More specifically, both $\lambda_{1}$ and $\lambda_{3}$ are almost constant with values $\lambda_{1} \approx 5$ and $\lambda_{3} \approx 4$ respectively, whereas $\lambda_{2}$ increases from 4 to 5 with $\sigma_{2}$. These behaviors are understood from the fact that $\pi_{\mathrm{SVS}}$ shrinks each singular value separately [14]. For $\pi_{\mathrm{S}}, \lambda_{1}$ is larger than $n=5$ when $\sigma_{2} \leq 8$ and thus the estimator is not minimax. This is compatible with Proposition 4.15. However, the sum $\lambda_{1}+\lambda_{2}+\lambda_{3}$ of eigenvalues, which is equal to the Frobenius risk $\mathrm{E}_{M}\left[\|\hat{M}-M\|_{\mathrm{F}}^{2}\right]=\operatorname{tr} R(M, \hat{M})$, does not exceed $n p=15$, because $\pi_{\mathrm{S}}$ is superharmonic in usual sense. This is similar to the fact that the James-Stein estimator is not minimax componentwise, even though it is minimax under the quadratic loss for the whole vector [12].
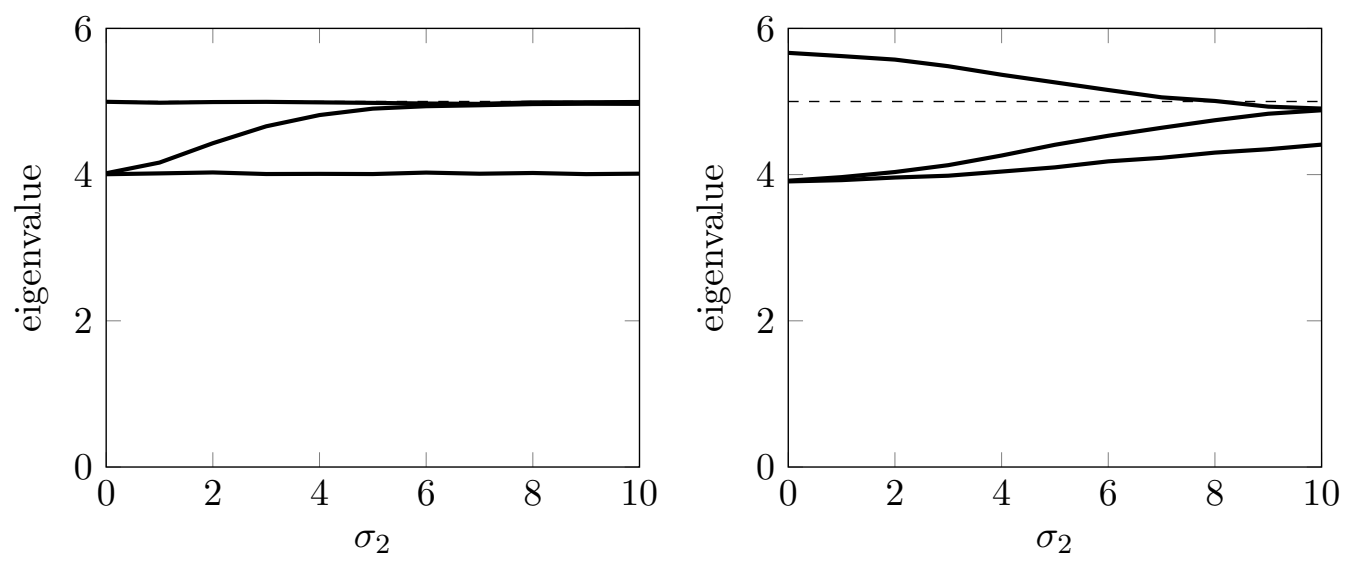

Figure 1: Eigenvalues of matrix quadratic risk of generalized Bayes estimators $(n=5, p=3$, $\left.\sigma_{1}=10, \sigma_{3}=0\right)$. left: $\pi_{\mathrm{SVS}}$. right: $\pi_{\mathrm{S}}$. The dashed line shows $n=5$.

Figure 2 plots the three eigenvalues $\lambda_{1} \geq \lambda_{2} \geq \lambda_{3}$ of the matrix quadratic risk with respect to $\sigma_{1}$ when $\sigma_{2}=\sigma_{3}=0$. Thus, the rank of $M$ is one. For $\pi_{\mathrm{SVS}}$, both $\lambda_{2}$ and $\lambda_{3}$ are almost constant around 4 , whereas $\lambda_{1}$ increases from 4 to 5 with $\sigma_{1}$. It indicates that $\pi_{\text {SVS }}$ works particularly well when $M$ has low rank. For $\pi_{\mathrm{S}}$, all eigenvalues are fairly small when $\sigma_{1}=0$, namely $M=O$. However, $\lambda_{1}$ increases rapidly with $\sigma_{1}$ and becomes larger than $n=5$ when $\sigma_{1} \geq 4$.

Next, we compare the Efron-Morris estimator $\hat{M}_{\mathrm{EM}}=X\left(I-(n-p-1)\left(X^{\top} X\right)^{-1}\right)$ and the James-Stein estimator $\hat{M}_{\mathrm{JS}}=\left(1-(n p-2) /\|X\|_{\mathrm{F}}^{2}\right) X$ in higher dimension. Note that these estimators have almost the same risk with the generalized Bayes estimators with respect to 

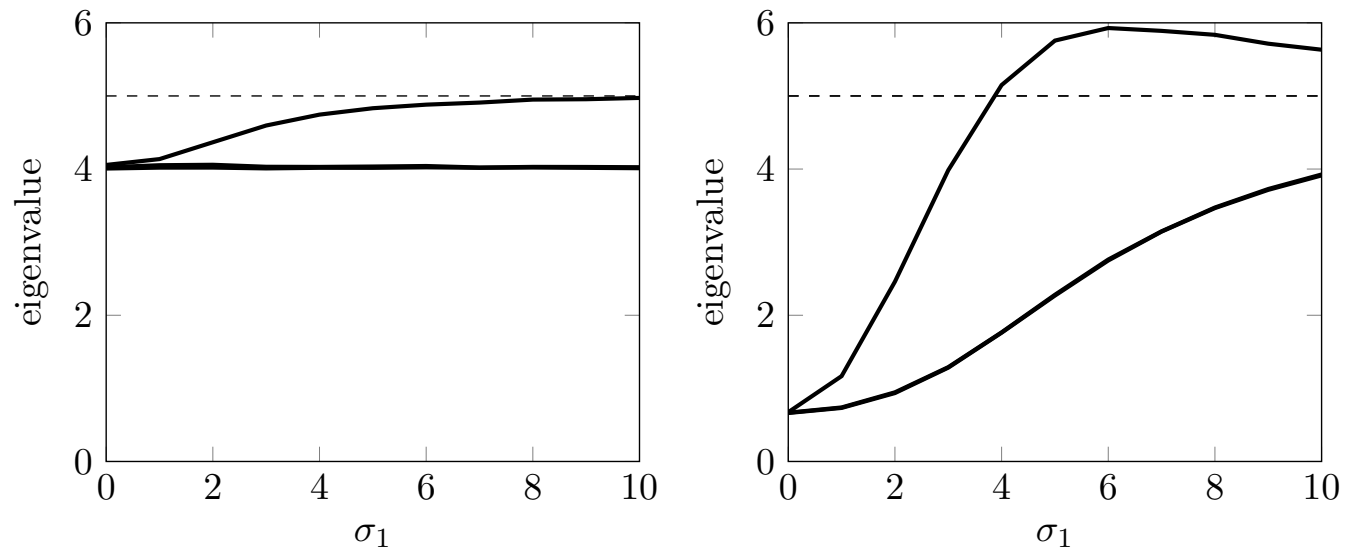

Figure 2: Eigenvalues of matrix quadratic risk of generalized Bayes estimators $(n=5, p=3$, $\left.\sigma_{2}=\sigma_{3}=0\right)$. left: $\pi_{\mathrm{SVS}}$. right: $\pi_{\mathrm{S}}$. The dashed line shows $n=5$. Note that the second and third eigenvalues almost overlap in both plots.

$\pi_{\mathrm{SVS}}$ and $\pi_{\mathrm{S}}$, respectively.

Figure 3 plots the 20 eigenvalues $\lambda_{1} \geq \cdots \geq \lambda_{20}$ of the matrix quadratic risk with respect to $\sigma_{1}$ when $n=100, p=20, \sigma_{i}=(6-i) / 5 \cdot \sigma_{1}(i=2, \ldots, 5)$ and $\sigma_{6}=\cdots=\sigma_{20}=0$. Thus, the rank of $M$ is five. The results are qualitatively the same with Figure 2, with the advantage of the singular value shrinkage more pronounced. For $\hat{M}_{\mathrm{EM}}$, all eigenvalues are smaller than $n=100$. In particular, the bottom 15 eigenvalues are almost constant around $20: \lambda_{6} \approx \cdots \approx \lambda_{20} \approx 20$. On the other hand, for $\hat{M}_{\mathrm{JS}}$, the largest eigenvalue $\lambda_{1}$ grows rapidly with $\sigma_{1}$ and exceeds $n=100$ when $\sigma_{1} \geq 10$. Other eigenvalues also increase with $\sigma_{1}$, including $\lambda_{6}, \ldots, \lambda_{20}$. These results show that the singular value shrinkage works well for low rank matrices.
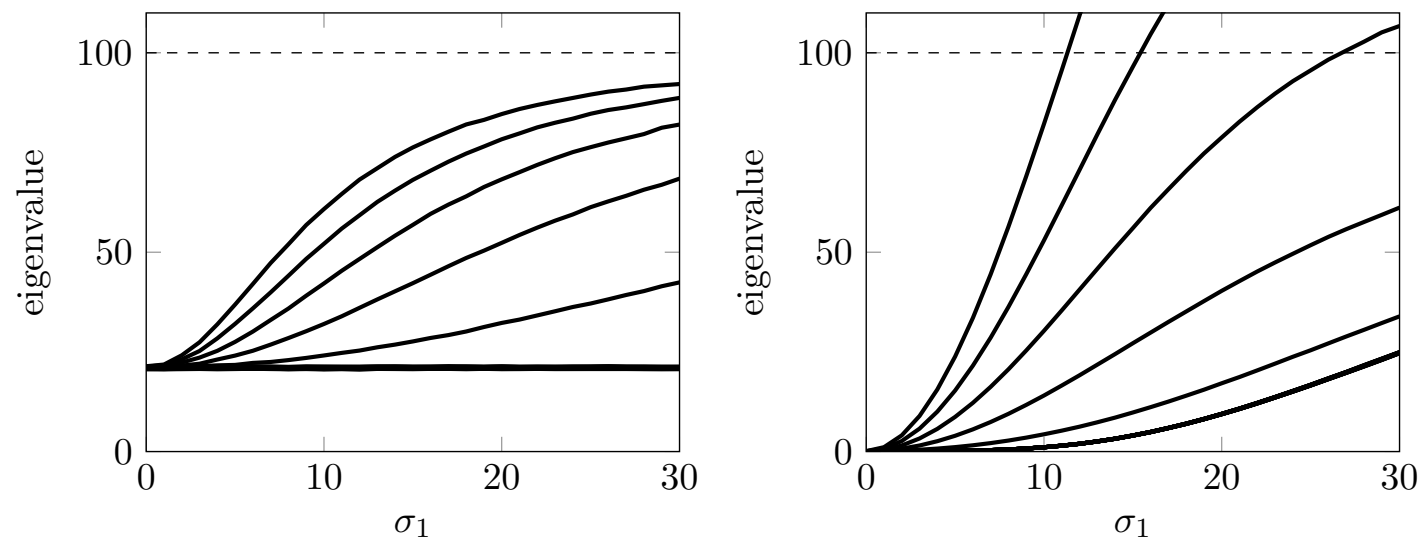

Figure 3: Eigenvalues of matrix quadratic risk $\left(n=100, p=20, \sigma_{i}=(6-i) / 5 \cdot \sigma_{1}\right.$ for $i=2, \ldots, 5$ and $\left.\sigma_{6}=\cdots=\sigma_{20}=0\right)$. left: Efron-Morris estimator. right: James-Stein estimator. The dashed line shows $n=100$. Note that the bottom 15 eigenvalues almost overlap in both plots.

For the Efron-Morris estimator, the above simulation results suggest that the $i$-th eigen- 
value $\lambda_{i}$ of the matrix quadratic risk depends only on the $i$-th singular value $\sigma_{i}$ of $M$ approximately: $\lambda_{i} \approx g_{n, p}\left(\sigma_{i}\right)$ for some function $g_{n, p}$. Finally, we investigate this functional relation $g_{n, p}$ numerically. Figure 4 plots $\lambda_{1}$ with respect to $\sigma_{1}$ when $\sigma_{2}=\cdots=\sigma_{p}=0$ for several values of $n$ and $p$. It indicates that $g_{n, p}(0) \approx p$, which is compatible with $R\left(O, \hat{M}_{\mathrm{EM}}\right)=(p+1) I_{p}$ from Corollary 2.6. In addition, Figure 4 implies $g_{n, p}(\sigma) \rightarrow n$ as $\sigma \rightarrow \infty$. This is understood from the fact that the Efron-Morris estimator $\hat{M}_{\mathrm{EM}}$ becomes essentially the same with the maximum likelihood estimator $\hat{M}=X$, which has the constant risk $R(M, \hat{M})=n I_{p}$, in a direction of a very large singular value [17, 14]. These properties of $g_{n, p}$ provide a quantification of the advantage of the Efron-Morris estimator over the maximum likelihood estimator when $M$ has low rank. Namely, if $M$ has rank $r<p$, then $\sigma_{r+1}=\cdots=\sigma_{p}=0$ and thus $\lambda_{r+1}, \ldots, \lambda_{p}$ should be close to $g_{n, p}(0) \approx p$. Therefore, the reduction in the Frobenius risk is evaluated as

$$
n p-\operatorname{tr} R\left(M, \hat{M}_{\mathrm{EM}}\right) \geq n p-r n-(p-r) p=n p\left(1-\frac{r}{p}\right)\left(1-\frac{p}{n}\right) .
$$

Thus, the Efron-Morris estimator attains large risk reduction especially when either $p / r$ or $n / p$ is large.
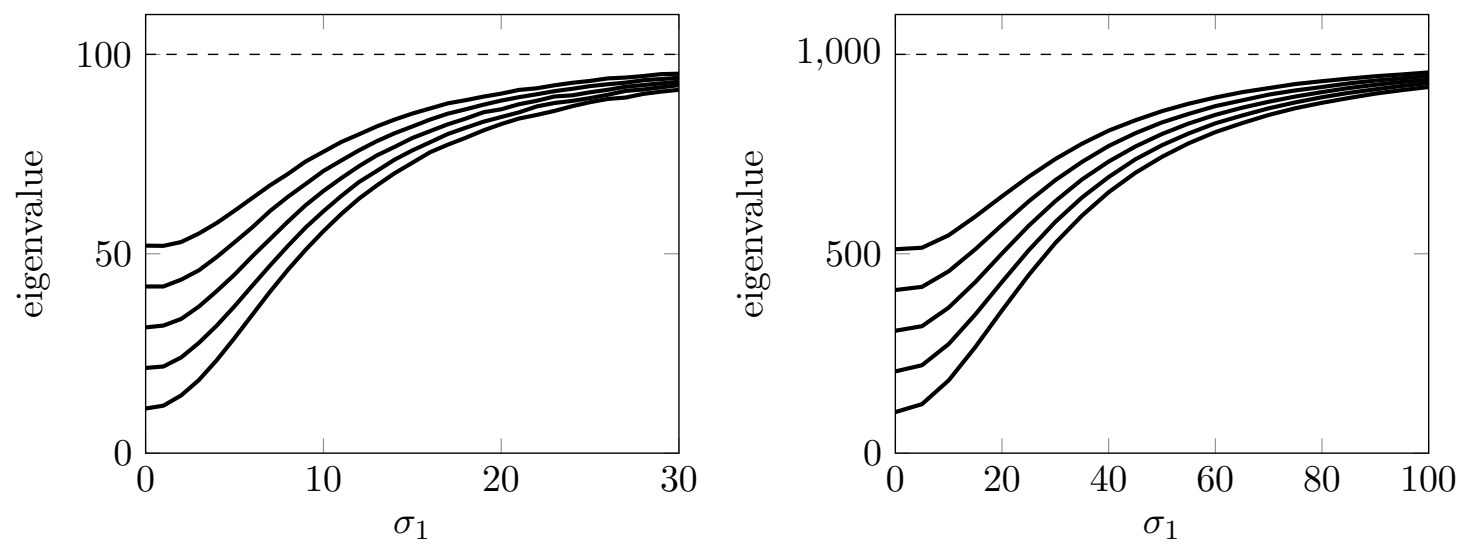

Figure 4: Largest eigenvalue of matrix quadratic risk of the Efron-Morris estimator $\left(\sigma_{2}=\right.$ $\left.\cdots=\sigma_{p}=0\right)$. left: $n=100, p=10,20, \ldots, 50$. right: $n=1000, p=100,200, \ldots, 500$. The dashed line shows $n$.

\section{Acknowledgements}

We thank Yuzo Maruyama for helpful comments. This work was supported by JSPS KAKENHI Grant Number 19K20220. This work was partially funded by grant \# 418098 from the Simons Foundation to William Strawderman.

\section{References}

[1] Abu-Shanab, R., Kent, J. T. \& Strawderman, W. E. (2012). Shrinkage estimation with a matrix loss function. Electronic Journal of Statistics 6, 2347-2355.

[2] Berman, A. \& Shaked-Monderer, N. (2003). Completely Positive Matrices. World Scientific. 
[3] Bilodeau, M. \& Kariya, T. (1989). Minimax estimators in the normal MANOVA model. Journal of Multivariate Analysis 28, 260-270.

[4] Efron, B. \& Morris, C. (1972). Empirical Bayes on vector observations: an extension of Stein's method. Biometrika 59, 335-347.

[5] Faith, M. (1993). Minimax Bayes estimators of a multivariate normal mean. Journal of Multivariate Analysis 8, 372-379.

[6] Fourdrinier, D., Strawderman, W. E. \& Wells, M. (2018). Shrinkage Estimation. New York: Springer-Verlag.

[7] George, E. I., Liang, F. \& Xu, X. (2006). Improved minimax predictive densities under Kullback-Leibler loss. Annals of Statistics 34, 78-91.

[8] Gupta, A. K. \& Nagar, D. K. (2000). Matrix Variate Distributions. New York: Chapman \& Hall.

[9] Helms, L. L. (2014). Potential Theory. New York: Springer-Verlag.

[10] Honda, T. (1991). Minimax estimators in the MANOVA model for arbitrary quadratic loss and unknown covariance matrix. Journal of Multivariate Analysis 36, 113-120.

[11] Komaki, F. (2006). Shrinkage priors for Bayesian prediction. Annals of Statistics 34, 808-819.

[12] Lehmann, E. L. \& Casella, G. (2006). Theory of Point Estimation. New York: Springer-Verlag.

[13] Magnus, J. R. \& Neudecker, H. (2019). Matrix Differential Calculus with Applications in Statistics and Econometrics. New York: Wiley.

[14] Matsuda, T. \& Komaki, F. (2015). Singular value shrinkage priors for Bayesian prediction. Biometrika 102, 843-854.

[15] Matsuda, T. \& Komaki, F. (2019). Empirical Bayes matrix completion. Computational Statistics \& Data Analysis 137, 195-210.

[16] Murota, K. (2003). Discrete Convex Analysis. Society for Industrial and Applied Mathematics.

[17] Stein, C. (1974). Estimation of the mean of a multivariate normal distribution. Proc. Prague Symp. Asymptotic Statistics 2, 345-381.

[18] XIE, M. (1993). All admissible linear estimates of the mean matrix. Journal of Multivariate Analysis 44, 220-226. 